Ann. Sci. forest., I975, $32(2)$, II3-I29.

\title{
INFLUENCE DES GROS RAYONS LIGNEUX SUR QUELQUES PROPRIÉTÉS DU BOIS DE HETRE
}

\author{
R. KELLER et F. THIERCELIN \\ avec la collaboration technique de J. R. PERrin \\ Station de Recherches sur la Qualité des Bois, \\ Centre national de Recherches forestières, I. N. R. A., \\ Champenoux, 54280 Seichamps
}

\section{RÉSUMÉ:}

Les gros rayons ligneux d'éprouvettes normalisées de hêtre (Fagus silvatica) ont été dénombrés et mesurés automatiquement par un analyseur de texture à partir de l'image photographique de leur plan tangentiel.

La comparaison des chiffres obtenus et des résultats des essais mécaniques et physiques exercés sur ces échantillons montre que les rayons sont des facteurs de forte résistance et de rétractibilité tangentielle élevée.

L'utilisation de la radiographie, au lieu de la photographie, permettrait d'obtenir encore une meilleure résolution par l'analyseur de texture; elle donne en outre le moyen d'évaluer, comme nous l'avons fait, la valeur propre de la densité (en $\mathrm{g} / \mathrm{dm}^{3}$ ) de ces éléments anatomiques, et ultérieurement de faire la part des propriétés qui leur sont liées et de celles qui sont régies par les autres éléments du bois.

\section{I. - INTRODUCTION}

Les rayons ligneux du bois sont présents chez toutes les essences, mais leur type, leur abondance et leurs dimensions varient de 1'une à 1'autre. Constitués de bandes radiales de cellules de parenchyme, auxquelles se mêlent chez certains résineux (pins, épicéas, douglas, etc.) des trachéides transversales, ils se lignifient en vieillissant.

Ils communiquent avec les autres éléments anatomiques grâce aux ponctuations des champs de croisement et, comme ils sont d'orientation perpendiculaire au fil du bois, permettent des transferts horizontaux de substances; ils accumulent des réserves avant chaque interruption de cycle végétatif qu'ils restituent au début du cycle suivant. 
Selon les espèces, les rayons ligneux peuvent constituer de 3 p. IOO à 30 p. IOO environ du volume de l'ensemble des éléments ligneux (A. J. C. PANshin et al., I964; R. Wagenfuhr, Ig66). Pour le hêtre, des chiffres moyens de l'ordre de 25 p. roo chez de jeunes plants et de I $_{5}$ p. Ioo chez les arbres adultes peuvent être retenus (C. Koltzenburg, ig66; G. Jayme et F. K. Azzola, I965).

Les rares études qui leur ont été plus particulièrement consacrées ont permis de préciser certains des domaines où ils jouent un rôle. Leurs cellules sont beaucoup plus courtes que les autres cellules longitudinales du bois et ne présentent pas les mêmes qualités papetières : leur masse spécifique est généralement élevée ( $\mathrm{F}$. W. TAYLOR, I 969 ; F. W. TAYLOR et T. E. WoOTEN, I973) et leur proportion est en liaison positive avec la dureté, la résistance en compression axiale et transversale (J. E. MYER, I922) ; ils contribuent à augmenter la charge maximale à la limite élastique, ainsi que le module d'élasticité radiale (R. W. Kenneny, ig68). De plus, ils constituent un facteur important d'anisotropie (A. P. Schniewind, I959 et ig66; J. B. Boutelje, I962). Certains rayons anormaux déprécient la qualité du bois (H. SACHSSE, I974). Leur plus ou moins grande abondance n'est pas le fait du hasard, mais est contrôlée génétiquement (E. TELLERup et S. de MuckadELL, I953).

L'objet de cette note est de présenter quelques observations faites sur des propriétés physiques et mécaniques d'échantillons de hêtre (Fagus silvatica) en liaison avec la quantité ou les dimensions des gros rayons dans leur plan ligneux.

On sait que chez le hêtre coexistent à la fois de petits et de gros rayons ligneux. Seuls ces derniers ont été pris en compte pour des raisons qui seront indiquées cidessous.

\section{2. - MATÉRIEL E'T MÉTHODE \\ 2. I. - Description sommaire des échantillons \\ et des essais mécaniques et physiques effectués}

Soixante échantillons parallélépipédiques de $2 \mathrm{~cm} \times 2 \mathrm{~cm} \times 6 \mathrm{~cm}$ ont été étudiés; ils ont été tirés chacun d'une éprouvette normalisée choisie aussi parfaitement de droit fil que possible et dont les faces tangentielles étaient bien perpendiculaires aux rayons. Les éprouvettes provenaient de quatre peuplements dont deux seulement assez abondamment représentés :

\begin{tabular}{l|c}
\hline $\begin{array}{l}\text { Origine } \\
\text { forêt de : }\end{array}$ & Nombre d'éprouvettes \\
\hline Epinal & 30 \\
Gérardmer & 25 \\
Retz & 3 \\
Haye & 2 \\
& \\
\hline Épinal (88), Gérardmer (88), Retz $(02)$, Haye (54).
\end{tabular}

Avant le dénombrement des rayons, ces éprouvettes ont subi diverses mesures physiques et essais mécaniques : 


\begin{tabular}{|c|c|c|}
\hline Type de mesure & $\begin{array}{l}\text { Abréviation } \\
\text { dans la suite } \\
\text { du texte }\end{array}$ & Unités \\
\hline 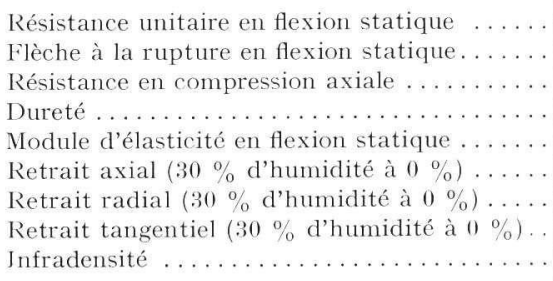 & $\begin{array}{l}\text { RFS } \\
\text { FLE } \\
\text { RCA } \\
\text { DUR } \\
\text { MOE } \\
\text { RAX } \\
\text { RRA } \\
\text { RTG } \\
\text { IDE }\end{array}$ & $\begin{array}{l}\mathrm{kg} / \mathrm{cm}^{2} \\
\mathrm{~mm} \\
\mathrm{~kg} / \mathrm{cm}^{2} \\
\text { arbitraire } \\
\mathrm{kg} / \mathrm{cm}^{2} \\
\% \\
\% \\
\% \\
\% \\
\mathrm{~g} / \mathrm{dm}^{3}\end{array}$ \\
\hline
\end{tabular}

\section{2. - Méthode de la mesure des variables caractérisant les gros rayons ligneux}

Les échantillons destinés à l'étude des rayons ont été photographiés suivant une face tangentielle de façon que l'ensemble de l'expérience figure sur une même plaque de $64 \mathrm{~mm} \times 89 \mathrm{~mm}$ (fig. I). C'est cette dernière qui a été analysée à l'aide d'un appareil Microvideomat de Zeiss (fig. 2) capable, dans certaines conditions, de trier et de compter des éléments contenus dans un ensemble lorsqu'ils sont mis en évidence par un moyen quelconque (éclairage, coloration, etc.). Dans le cas de cette expérience, les gros rayons ligneux apparaissent sur la plaque comme des

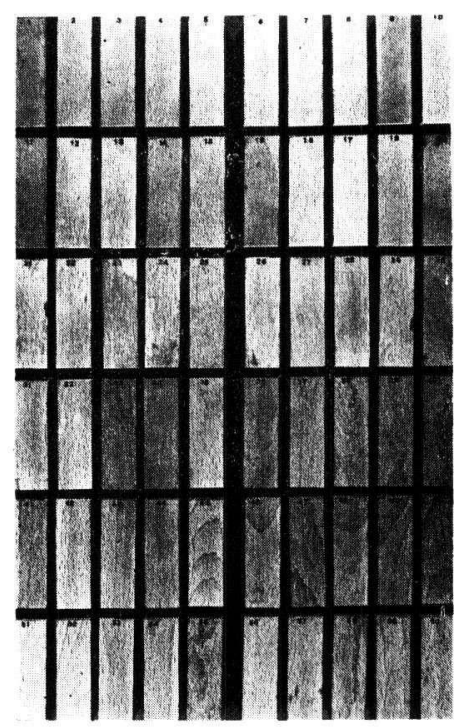

Fic. I. - Positif de la plaque photographique

à partir de laquelle ont été déterminés les pourcentages de gros rayons ligneux

Les dimensions de la face tangentielle des échantillons sont:

longueur $=6 \mathrm{~cm} ; \quad$ largeur $=3 \mathrm{~cm}$

Positive print of the photographic plate on rehich the percentages of big wood rays were measured

Dimensions of tangential face of samples:

$$
\text { length }=6 \mathrm{~cm} ; \quad \text { width }=2 \mathrm{~cm}
$$


taches elliptiques claires sur un fond sombre, alors que les petits rayons ne s'y individualisent pas nettement.

L'appareil analyseur est constitué d’un microscope surmonté d'une caméra couplée à un écran de télévision. L'objet étudié est placé sous le microscope et le choix des éléments à mesurer se fait d'après l'image vue sur l'écran; leur sélection est fonction de la gamme des tons de gris que l'appareil peut définir (l'idéal serait de pouvoir faire correspondre un ton à un type d'élément), et plus le contraste entre la zone à mesurer et le reste est grand, meilleure est la précision de la détermination de cette zone.

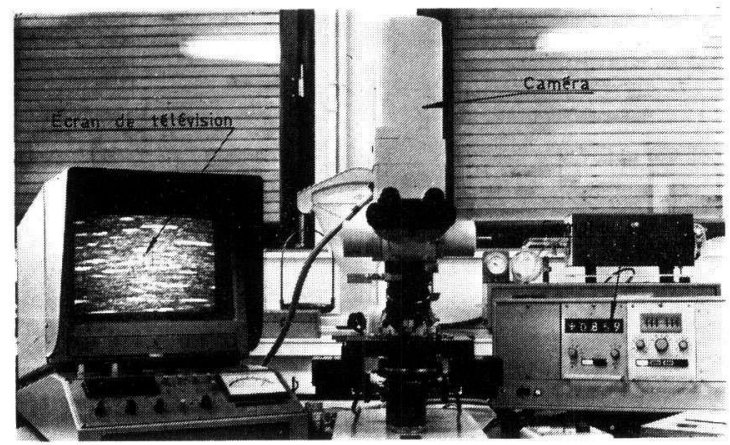

FIG. 2. - Dispositif de mesure utilise

- au centre, le microscope surmonté de la caméra

- à gauche, l'écran de télévision où apparaissent les rayons ligneux (taches claires) à mesurer

- à droite, le compteur (il indique ici 8,59 p. Ioo de rayons par rapport à la surface totale mesurable de l'écran).

\section{Measuring device used}

— in the middle, the microscope and, above, the camera

- on the left, the television screen on which the wood rays appear (light areas)

- on the right, the counter (here indicating $8,59 \mathrm{p}$. roo of wood rays with respect to the total measurable area of the screen).

Pour évaluer la proportion en volume des gros rayons, on a supposé que le rapport $\frac{\text { volume des rayons ligneux }}{\text { volume total de bois }}$ était le même que $\frac{\text { surface tangentielle des rayons ligneux }}{\text { surface tangentielle totale analysée }}$.

Du fait que seuls les gros rayons étaient suffisamment contrastés par rapport au reste, les proportions trouvées sont plus faibles que les chiffres cités dans la littérature qui englobent les gros et les petits rayons du hêtre, mais elles peuvent néanmoins servir à des comparaisons entre les soixante éprouvettes que nous avons utilisées puisque, pour elles, elles sont exactes en valeur relative.

Le pourcentage et le nombre de gros rayons indiqués pour chacun des échantillons sont égaux à la moyenne de six mesures. Chaque mesure correspond, étant donné le rapport entre le nombre de lignes de l'écran de télévision et les caractéristiques du microscope et de la caméra, à l'exploration de $7,8 \mathrm{~mm}^{2}$ de la plaque photographique, soit, en définitive, à 2 Io mm² environ de surface tangentielle de bois (12,36 $\mathrm{mm} \times 17,00 \mathrm{~mm}$ ).

\section{3. - RÉSULTATS}

Les résultats sont résumés dans le tableau I où trois nombres caractérisent les gros rayons ligneux, leur pourcentage (PRL), leur nombre au $\mathrm{cm}^{2}$ (NRL), leur dimension exprimée par le rapport $\begin{gathered}\text { pourcentage } \\ \text { nombre au } \mathrm{cm}^{2}\end{gathered}$

(I)RL) qui indique le pourcentage moyen représenté par un gros rayon ligneux. 
Ces chiffres n'appellent pas de commentaires particuliers, sinon que la dureté a été évaluée dans une unité arbitraire et que, plus le nombre la caractérisant est grand, plus elle est faible.

TABLEAU I

Valeurs caractérisant les douze variables étudiées

Values characterizing the twelve investigated features

\begin{tabular}{|c|c|c|c|c|c|}
\hline Variable & Minimum & Maximum & Moyenne & Écart-type & $\begin{array}{c}\text { Coefficient } \\
\text { de variation }(\%)\end{array}$ \\
\hline $\begin{array}{l}\text { RFS }\left(\mathrm{kg} / \mathrm{cm}^{2}\right) \\
\text { FLE }(\mathrm{mm}) \\
\text { RCA }\left(\mathrm{kg} / \mathrm{cm}^{2}\right) \\
\text { DUR (unité arbitraire) } \\
\text { MOE }\left(\mathrm{kg} / \mathrm{cm}^{2}\right) \\
\text { RAX }(\%) \\
\text { RRA }(\%) \\
\text { RTG }(\%) \\
\text { IDE }\left(\mathrm{g} / \mathrm{dm}^{3}\right) \\
\text { PRL }(\%) \\
\text { NRL }\left(\mathrm{nombre} / \mathrm{cm}^{2}\right) \\
\text { DRL }(\%)\end{array}$ & $\begin{array}{c}988 \\
8,6 \\
406 \\
43 \\
1060000 \\
-\quad 0,1 \\
2,3 \\
6,8 \\
432 \\
2,62 \\
16,4 \\
0,06\end{array}$ & $\begin{array}{c}1432 \\
13,8 \\
754 \\
65 \\
181000 \\
0,8 \\
4,2 \\
12,4 \\
537 \\
8,55 \\
49,8 \\
0,22^{\prime}\end{array}$ & $\begin{array}{c}1180 \\
10,9 \\
542 \\
53 \\
135000 \\
0,25 \\
3,3 \\
9,5 \\
486 \\
4,31 \\
30,5 \\
0,145\end{array}$ & $\begin{array}{c}120 \\
1,1 \\
60 \\
6,4 \\
18000 \\
0,2 \\
0,4 \\
1,5 \\
26 \\
1,24 \\
9,5 \\
0,036\end{array}$ & $\begin{array}{r}10,2 \\
10,1 \\
11,1 \\
12,1 \\
13,3 \\
80 \\
12,1 \\
15,8 \\
5,4 \\
28,8 \\
31,2 \\
2 / 4,8\end{array}$ \\
\hline $\begin{array}{l}\text { moyenne }=\text { mean } \\
\text { écart-type = standar } \\
\text { coefficient de varia } \\
\mathrm{RFS}=\text { maximum loc } \\
\begin{aligned} \mathrm{RLE}= & \text { maximum } \\
& \text { bending } \\
\mathrm{RCA}= & \text { maximum lo } \\
& \text { rallel to grain }\end{aligned}\end{array}$ & $\begin{array}{l}\text { deviation } \\
\text { ion }=\text { coef } \\
\text { lin static ber } \\
\text { eflection in } \\
\text { in compre: }\end{array}$ & $\begin{array}{l}\text { nt of } \\
\text { iation } \\
\text { ig } \\
\text { static } \\
\text { n pa- }\end{array}$ & \multicolumn{3}{|c|}{$\begin{array}{l}\mathrm{DUR}=\text { hardness } \\
\mathrm{MOE}=\text { modulus of elasticity } \\
\mathrm{RAX}=\text { axial shrinkage } \\
\mathrm{RRA}=\text { radial shrinkage } \\
\mathrm{RTG}=\text { tangential shrinkage } \\
\mathrm{IDE}=\text { specific gravity } \\
\mathrm{PRL}=\text { percentage of wood rays } \\
\mathrm{NRL}=\text { number of wood rays per } \mathrm{cm}^{2} \\
\mathrm{DRL}=\text { mean dimension of one wood ray }\end{array}$} \\
\hline
\end{tabular}

Huit variables sont considérées comme " à expliquer "; ce sont les résultats des essais mécaniques et des mesures de retrait. Trois variables sont considérées comme " explicatives "; il s'agit des données mesurées sur les rayons ligneux. L'infradensité joute un rôle double : explicative vis-à-vis des caractères physiques et mécaniques, et expliquée vis-à-vis des rayons.

\section{I. - L'infradensité considérée comme variable explicative}

Comme l'on pouvait s'y attendre, les calculs de corrélation ont généralement fait ressortir l'infradensité comme la meilleure variable explicative des résultats physiques et mécaniques. Le tableau 2 montre qu'elle est particulièrement bien liée à la résistance en flexion statique, à la résistance en compression axiale, à la dureté puisque $|r|>0,700$. Elle est en corrélation également avec le module d'élasticité, le retrait radial et le retrait tangentiel. 


\section{2. Les rayons lignenx et l'infradensité}

Deux des trois variables caractérisant les rayons ligneux : le pourcentage et le nombre au $\mathrm{cm}^{2}$, ont donné avec l'infradensité des coefficients moyennement élevés, respectivement $r=0,28 \mathrm{I}$ et $r=0,344$. Si le pourcentage et le nombre des rayons par unité de surface semblent être des facteurs liés à une densité du bois forte, en revanche leur dimension moyenne n'apparaît pas ici comme une variable explicative significative.

\section{3. - Corrélations entre les variables relatives aux rayons}

Le nombre de rayons au $\mathrm{cm}^{2}$ est lié positivement au pourcentage $(r=0,6 \mathrm{I} 6)$ et négativement à la dimension $(r=-0,565)$, probablement en grande partie à cause de la façon dont a été définie cette dernière variable.

Le pourcentage n'est pas lié à la dimension, ce qui indique que chez les échantillons mesurés, un fort volume de rayons est dî à la présence d'un nombre élevé de rayons plutôt qu'à une forte dimension moyenne de ces éléments.

\section{4. - Les chiffres relatifs \\ aux rayons ligneux considérés comme variables explicatives}

Bien que dans cette étude, toute la part représentée par les petits rayons n'ait pu être mesurée, ce qui nous a conduits à adopter comme pourcentage moyen le chiffre très faible de 4,3I p. IOO (tabl. I), des indications ont pu être dégagées sur le rôle de ces tissus dans les propriétés du bois.

\section{4I. Le pourcentage.}

On a déjà vu qu'il est en corrélation positive avec l'infradensité, mais c'est la seule de toutes les variables explicatives à être liée à la flèche à la rupture en flexion statique. Il a donné un coefficient significatif au seuil de I p. Ioo avec la dureté et au seuil de I p. I ooo avec le retrait tangentiel.

\section{42. Le nombre an $\mathrm{cm}^{2}$.}

Les liaisons avec les caractéristiques mécaniques, résistance en flexion statique, résistance en compression axiale, dureté et module d'élasticité sont significatives au seuil de 5 p. Ioo.

Les coefficients de corrélation avec le retrait radial et le retrait tangentiel valent respectivement 0,347 et 0,598 . Ce dernier chiffre est significatif à I p. I ooo et dépasse ce que donne l'infradensité avec la même variable. Pour le retrait tangentiel, c'est donc l'abondance des gros rayons à l'unité de surface qui constitue la meilleure variable explicative.

\section{43. La dimension.}

Dans les cas de la résistance en compression axiale, du module d'élasticité et du retrait radial où le nombre au $\mathrm{cm}^{2}$ donne des coefficients significatifs positifs, la dimension donne des coefficients négatifs, à peu près équivalents en valeur absolue. Notons cependant que, malgré sa définition, elle n'est liée ni à la résistance à la flexion statique, ni à la dureté, ni au retrait tangentiel, alors que le nombre au $\mathrm{cm}^{2}$ l'est. 
I1 est plus intéressant de constater que la dimension est la meilleure variable explicative du retrait radial, avec un coefficient de corrélation de - o,388 significatif au seuil de I p. IOo, dépassant en valeur absolue ce que donne l'infradensité. Le retrait radial est d'autant plus faible que la dimension unitaire moyenne des gros rayons est plus forte.

\section{5. - Comparaison sur la valeur de l'infradensité et les données relatives aux rayons en tant que variables explicatives}

On connaît, et les chiffres du tableau 2 permettent de le vérifier, la valeur de la densité du bois comme estimateur de ses propriétés; effectivement, dans la plupart des cas, c'est le chiffre d'infradensité qui a donné les coefficients de corrélation les plus significatifs. Mais, dans trois cas, les caractéristiques des rayons se sont révélées être plus discriminantes qu'elle dans la recherche des liaisons ; ces trois cas se partagent entre les trois types de variables:

La proportion de rayons est en corrélation avec la flèche à la rupture, alors que l'infradensité ne donne rien.

\section{TABLEAU 2}

Coefficients de corrélation entre les neuf variables à expliquer et les quatre variables explicatives

Correlation coefficients between the nine variables " to be explained" and the four explanatory variables

\begin{tabular}{|c|c|c|c|c|c|c|c|c|}
\hline \multirow{3}{*}{$\begin{array}{l}\text { IDE } \\
\text { PRL } \\
\text { NRL } \\
\text { DRL }\end{array}$} & RFS & FLE & \multicolumn{2}{|r|}{ RCA } & \multicolumn{2}{|c|}{ DUR } & MOE & RAX \\
\hline & $\begin{array}{l}0,817^{* * *} \\
0,239 \text { NS } \\
0,317^{*} \\
-0,177 \text { NS }\end{array}$ & $\begin{array}{l}0,247 \text { NS } \\
0,262^{*} \\
0,173 \text { NS } \\
0,102 \text { NS }\end{array}$ & - & $\begin{array}{l}0,707 * * * \\
0,162 \mathrm{NS} \\
0,308^{*} \\
0,305^{*}\end{array}$ & $\begin{array}{r}-0, \\
-0, \\
-0, \\
0,\end{array}$ & & $\begin{array}{c}0,392 * * \\
0,086 \mathrm{NS} \\
0,311^{*} \\
-0,304 *\end{array}$ & $\begin{array}{r}-0,179 \mathrm{NS} \\
0,017 \mathrm{NS} \\
0,164 \mathrm{NS} \\
-0,111 \mathrm{NS}\end{array}$ \\
\hline & RRA & RTC & & II & & & PRL & NRL \\
\hline $\begin{array}{l}\text { IDE } \\
\text { PRL } \\
\text { NRL } \\
\text { DRL }\end{array}$ & $\begin{array}{c}0,364 * * \\
0,037 \mathrm{NS} \\
0,347 * * \\
-0,388 * *\end{array}$ & $\begin{array}{r}0,57 \\
0,48 \\
0,59 \\
-0,22\end{array}$ & & $\begin{array}{r}0,2 \\
0,3 \\
-0,1\end{array}$ & $\begin{array}{l}1 * \\
4 * * \\
4 \mathrm{NS}\end{array}$ & & $\begin{array}{c}1 \\
0,616 * * * \\
0,252 \mathrm{NS}\end{array}$ & $\begin{array}{c}1 \\
-0,565 * * *\end{array}$ \\
\hline
\end{tabular}

Seuils de signification :

$$
\begin{array}{rll}
* & : 5 \text { p. } 100 \quad r=0,254 \\
* * & : 1 \text { p. } 100 \quad r=0,330 & \\
* * * & : 1 \text { p. } 1000 \quad r=0,41 ' t \\
\text { NS : coefficient non significatif. } & \\
*: & 5 \text { p. } 100 \text { confidence level } & \mathrm{r}=0,254 \\
* *: 1 \text { p. } 100 \text { confidence level } & \mathrm{r}=0,330 \\
* * *: 1 \text { p. } 1000 \text { confidence level } & \mathrm{r}=0,414 \\
\text { NS : value not significant. } &
\end{array}
$$


Le nombre de rayons donne le meilleur coefficient avec le retrait tangentiel; il en est de même pour la dimension des rayons et le retrait radial.

L'action des rayons, mesurés par leur proportion ou leur nombre, sur les propriétés du bois est en bon accord avec ce que donne la littérature. La notion de dimension est également utile à prendre en considération puisqu'elle fournit sa part dans une meilleure connaissance du retrait. Pour aller plus loin, peut-être faudrait-il lui adjoindre un nombre caractérisant la forme des rayons, du type rapport de la hauteur à la largeur, comme l'ont déjà fait certains auteurs dans une étude génétique des gros rayons de hêtre (E. TELLERUP et M. S. de MUCKadELL, op. cit.).

Seul le retrait axial n'a donné aucun coefficient de corrélation significatif. Les incertitudes dues à sa méthode de mesure ne sont certainement pas étrangères à cet état de choses.

\section{6. - Essais d'amélioration de l'explication des propriétés du bois par utilisation d'une méthode du type "régression progressive"}

Sept caractères physiques et mécaniques sont, on l'a vu, en liaison avec l'une des quatre variables explicatives. On a cherché dans quelle mesure pourrait être amélioré le pourcentage d'explication, pris égal à $r^{2}$, en calculant à nouveau les corrélations entre les variables à expliquer et des combinaisons linéaires de variables explicatives. Les résultats sont reportés an tableau 3 .

\section{6I. Propriétés mécaniques.}

Dans trois cas sur cinq, le résultat a été favorable, mais plus ou moins nettement. Aucune amélioration n'a été trouvée pour la résistance en flexion statique et la flèche qui peuvent donc se mettre sous la forme :

$$
\begin{aligned}
& \text { RFS }_{\mathrm{kg} / \mathrm{cm}^{2}}=-665+3,8 \mathrm{IDE}_{\mathrm{g} / \mathrm{dm}^{3}} \\
& \mathrm{FLE}_{\mathrm{mm}}=9,85+0,233 \mathrm{PRL}_{\%}
\end{aligned}
$$

L'explication de la résistance en compression axiale et du module d'élasticité est améliorée si l'on adjoint à l'infradensité la dimension des rayons ligneux. Dans le cas de la dureté, c'est le pourcentage qui doit être envisagé. Finalement, on peut écrire :

$$
\begin{aligned}
& \mathrm{RCA}_{\mathrm{kg} / \mathrm{cm}^{2}}=-\mathrm{I} 82,3+\mathrm{I}, 57 \quad \mathrm{IDE}_{\mathrm{g} / \mathrm{dm}^{3}}-27 \mathrm{I}, 7 \quad \mathrm{DRL}_{\mathrm{\%}} \\
& \mathrm{DUR}_{\text {unité arhitraire }}=\quad \mathrm{I} 4,59-0,0 \mathrm{I} 8 \mathrm{IDE}_{\mathrm{g} / \mathrm{dm}^{3}}-0,083 \mathrm{PRI}_{\%} \\
& \mathrm{MOE}_{\mathrm{kg} / \mathrm{cm}^{2}}=28000+250,5 \quad \mathrm{IDE}_{\mathrm{gg} / \mathrm{dm}^{3}}-\mathrm{IO} 4900 \mathrm{DRL}_{\%} \%
\end{aligned}
$$

\section{62. Retrait.}

Le retrait radial est mieux expliqué si on adjoint le pourcentage à la dimension. Ici donc l'infradensité n'entre pas en jeu dans l'équation.

Le retrait tangentiel voit son coefficient de corrélation passer de 0,573 à 0,709 si l'infradensité est associée au nombre de rayons. C'est ce dernier cas qui fournit l'amélioration la plus forte. Par suite, on peut écrire :

$$
\begin{aligned}
& \text { (I) } \quad \mathrm{RRA}_{\%}=3,84-4,85 \mathrm{DRL}_{\%}+0,04 \mathrm{PRL}_{\%} \\
& \text { (2) } \\
& \text { RTG } \%=-3, \mathrm{I} 7+0,8 \mathrm{I} 2 \mathrm{NRL}+0,0 \mathrm{I} 85 \mathrm{IDE}_{\mathrm{g} / \mathrm{dm}^{3}}
\end{aligned}
$$


TABLEAU 3

Coefficients de corrélation entre les variables physiques et mécaniques et, d'une part une variable explicative,

d'autre part une combinaison linéaive de deux variables explicatives

Correlation cofficients between the physical and mechanical variables and, on the one hand an explanatory variable on the other hand a linear combination of two explanatory variables

\begin{tabular}{|c|c|c|c|c|c|c|c|}
\hline & RFS & FLE & $\mathrm{RCA}$ & DUR & MOE & RRA & RTG \\
\hline $\begin{array}{c}\gamma_{1} \\
\text { variable explicative } \\
\% \text { expliqué }=r_{1}^{2}\end{array}$ & $\begin{array}{l}0,817 * * * \\
\mathrm{IDE} \\
66,5\end{array}$ & $\begin{array}{c}0,262^{*} \\
\text { PRL } \\
6,9\end{array}$ & $\begin{array}{l}0,707 * * * \\
\text { IDE } \\
50,0\end{array}$ & $\begin{array}{l}0,769 * * * \\
\text { IDE } \\
59,1\end{array}$ & $\begin{array}{l}0,392 * * \\
\text { IDE } \\
15,4\end{array}$ & \begin{tabular}{|c|}
$-0,388^{* *}$ \\
DRL \\
13,2
\end{tabular} & $\begin{array}{c}0,598^{* * *} \\
\text { NRL } \\
32,8\end{array}$ \\
\hline $\begin{array}{c}r_{\mathbf{2}}\left(\mathrm{si}\left|r_{2}\right|>\left|r_{1}\right|\right) \\
\text { variables explicatives } \\
\% \text { expliqué }=r_{2}^{2} \\
\text { gain }(\%)\end{array}$ & & & $\begin{array}{c}0,727 * * * \\
\mathrm{IDE}, \mathrm{DRL} \\
52,9 \\
2,9\end{array}$ & $\begin{array}{c}0,789 * * * \\
\text { IDE, PRL } \\
62,3 \\
3,2\end{array}$ & $\begin{array}{c}0,455^{* * *} \\
\text { IDE, DRL } \\
20,7 \\
5,3\end{array}$ & \begin{tabular}{|c|}
$0,411 * * *$ \\
DRL, PRL \\
16,9 \\
3,7
\end{tabular} & $\begin{array}{c}0,709 * * * \\
\text { NRL, IDE } \\
50,3 \\
17,5\end{array}$ \\
\hline
\end{tabular}

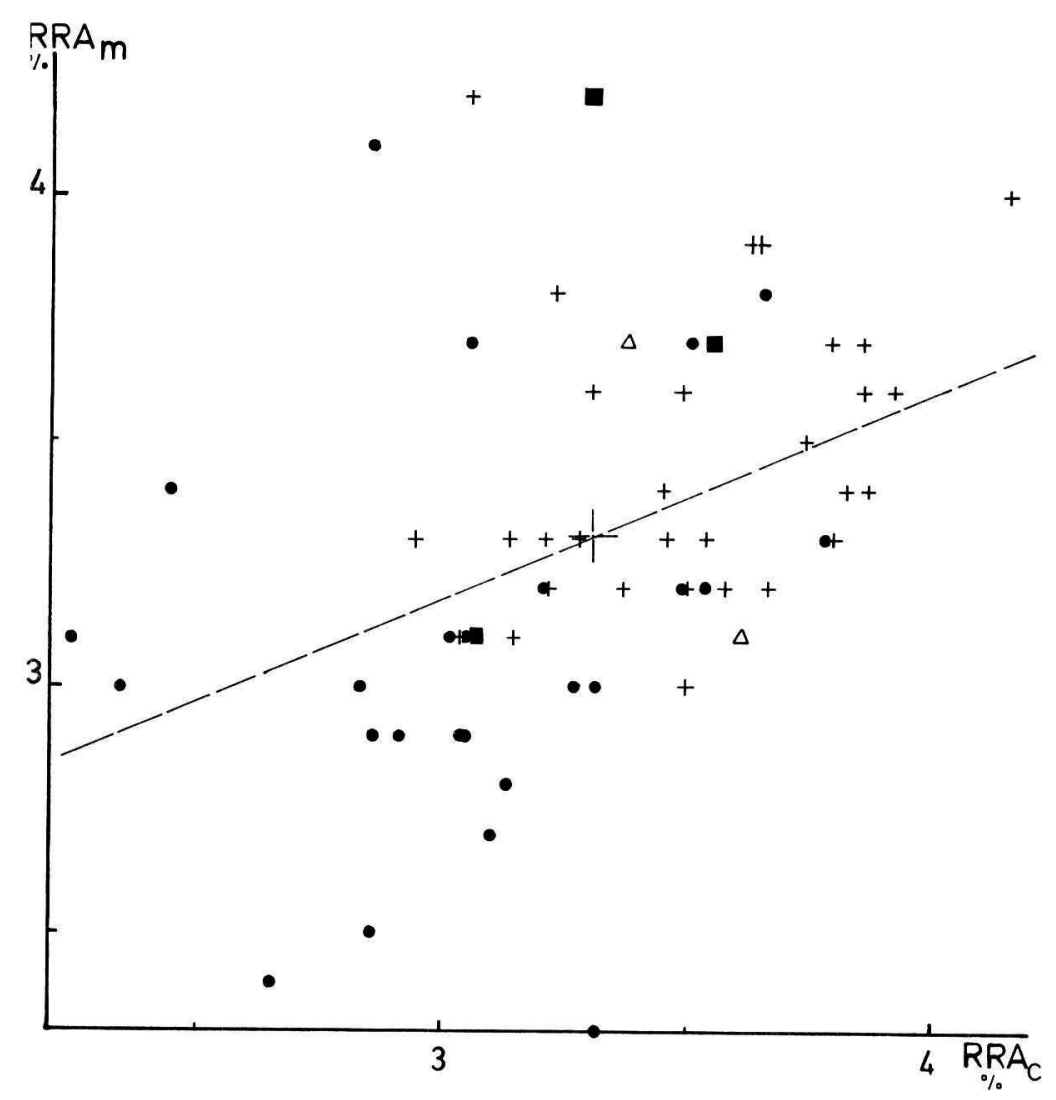

FIG. 3. - Graphique montrant la liaison entre le retrait radial mesuvé et le vetrait radial calculé par l'équation (1)

Graph showing the relationship between the measured radial shrinkage and that calculated by equation (1) 
Les graphiques des figures 3 et 4 mettent en évidence les liaisons existant entre les valeurs des variables mesurées portées en ordonnées et les valeurs prévisibles déduites des équations (I) et (2) portées, elles, en abscisses.

Les corrélations observées sont probablement significatives parce qu'on a utilisé des échantillons provenant de quatre peuplements différents, ce qui a eu pour effet d'étendre la gamme des retraits radial et tangentiel.

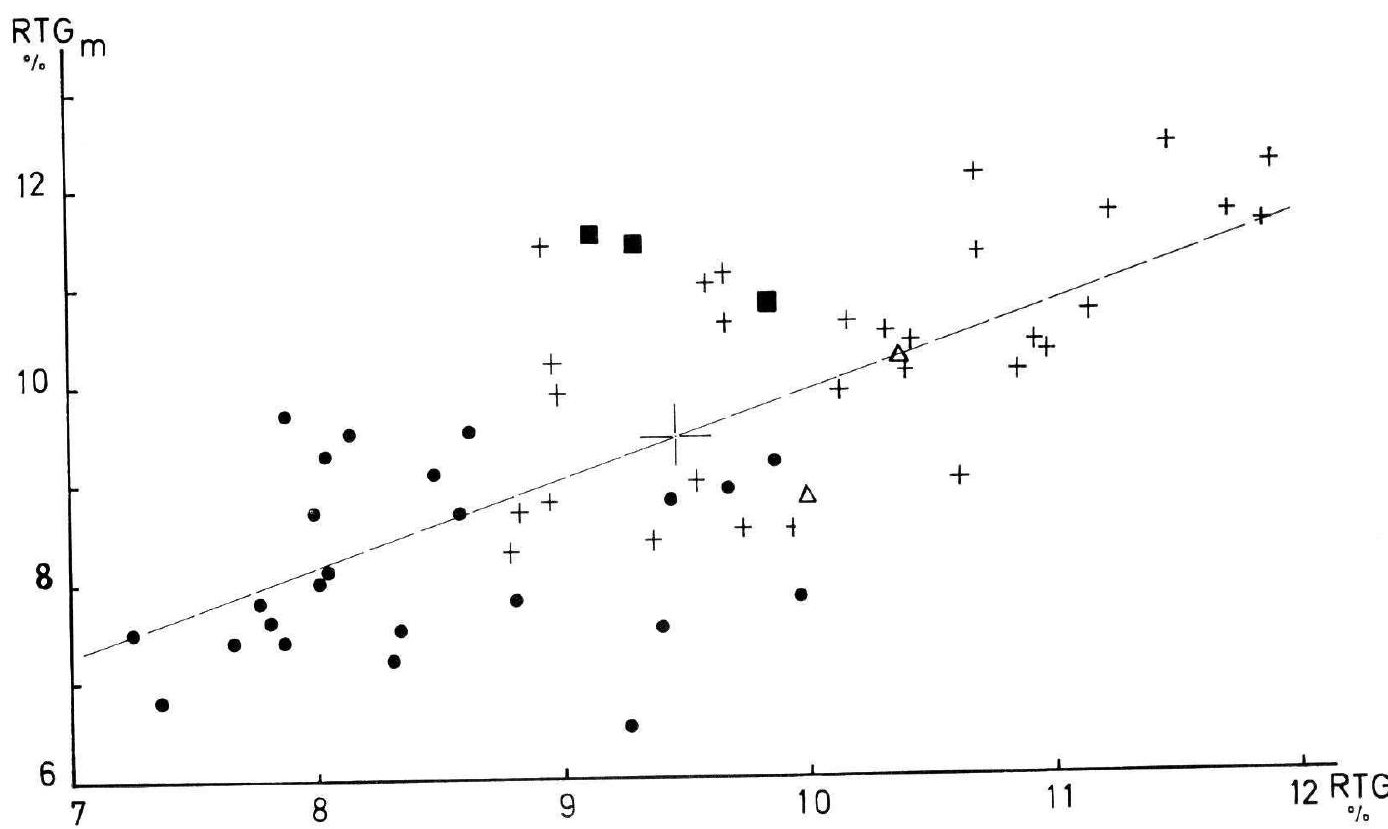

FIG. 4. - Graphique montrant la liaison entre le retrait tangentiel mesuré et le retrait tangentiel calculé par l'équation $\left(^{(2)}\right.$

Graph showing the relationship between the measured tangential shrinkage and that calculated by equation $\left({ }^{2}\right)$

Sur les graphiques ont été distinguées à dessein les quatre origines, les croix correspondant à Épinal, les points à Gérardmer, les carrés à Retz et les triangles à Haye. Mis à part ces deux dernières forêts, très faiblement représentées, Épinal et Gérardmer constituent deux nuages assez bien distincts et complémentaires. Le cas du retrait tangentiel est le plus net. Gérardmer a à la fois le plus faible retrait radial et le plus faible retrait tangentiel. Retz et Haye sont intermédiaires.

Comme, par ailleurs, nous n'avons pas de précisions sur les éprouvettes autres que leur origine géographique, il est difficile de donner une explication au fait de cette dissociation des valeurs en deux groupes correspondant aux origines. S'agit-il d'un influence de l'âge des arbres, du milieu, ou d'un phénomène à cause génétique? Il est impossible d'y voir plus clair avec ces seuls échantillons (E. TELLERUP et M. S. de Muckadeli, I953 op. cit. ; H. Polge et al., i972); 


\section{4. - MODIFICATIONS ET EXTENSIONS POSSIBLES DE LA MÉTHODE}

\section{I. - Critiques du procédé photographique employé}

Les valeurs absolues des chiffres trouvés sont très probablement trop faibles du fait que l'image photographique des échantillons présente des défauts pratiquement impossibles à éliminer. Ces défauts sont dus en partie à la difficulté qu'il y a à obtenir un éclairage uniforme de tous ces échantillons, même photographiés en bloc comme on l'a fait ; ils proviennent aussi du fait que, sur une même face tangentielle, peuvent affleurer plusieurs cernes et leurs limites, ce qui y fait apparaître des zones de couleurs différentes; enfin, des différences de teinte générale existent entre les éprouvettes. Le résultat est que seuls les gros rayons ont pu être mesurés.

Or, si l'on veut faire des études sur des échantillonnages abondants, cela fait presque nécessairement exclure les études anatomiques classiques et les dénombrements et mesures manuelles à partir de coupes microscopiques qui sont très longs, pour essayer d'obtenir des résultats automatiquement par des analyseurs de texture du genre de celui qui nous a servi. Ces appareils ont besoin de pouvoir différencier les éléments à mesurer et la difficulté est de trouver le moyen de les rendre suffisamment distincts les uns des autres, ou d'individualiser un élément par rapport à tout le reste.

\section{2. - Modification de la méthode dans le cas des rayons ligneux de hêtre}

$\mathrm{Si}$ on radiographie les échantillons au lieu de les photographier, toutes les difficultés dues à la couleur disparaissent (fig. 5). Lorsque les échantillons sont radiographiés radialement, les rayons $\mathrm{X}$ traversant perpendiculairement leurs faces tan-
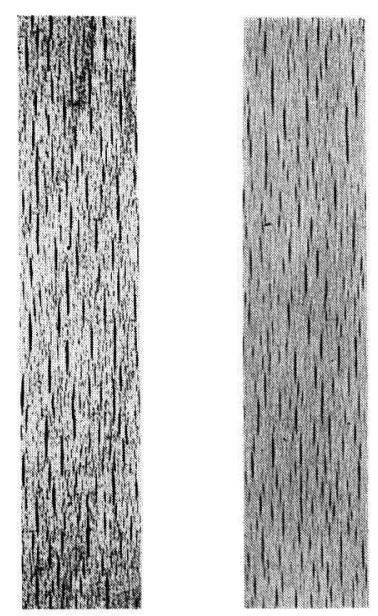

FIG. 5. - Photographie (à gauche) et positif de radiographie (à droite) de deux parties d'un même échantillon

Les petits et gros rayons ligneux ressortent mieux sur le fond uniforme de la radiographie

Photograph (on the left) and X-ray photograph positive print (on the right) of two parts of the same sample

The little and big wood rays stand out better against the uniform background of the radiograph Annales des Sciences forestières. -- 1975. 
gentielles, seules apparaissent sur le film des zones sombres, correspondant aux éléments anatomiques autres que les rayons, et des taches claires, images des petits et gros rayons. Dans ces conditions, 1'analyseur de texture dénombrera et mesurera avec une exactitude accrue les rayons ligneux, avec peut-être même la possibilité de distinguer les principales classes de dimensions.

Pour obtenir une bonne netteté de ces images, il faut scier les éprouvettes à une épaisseur inférieure ou égale à $3 \mathrm{~mm}$ et uniforme sur toute la surface à mesurer. Cette opération est faite avec des scies jumelées dont le plan peut être réglé de façon précise, aussi exactement perpendiculaire que possible à la direction radiale générale des rayons (F. THIERCELIN et J. R. PERrin, I972).

La radiographie a un autre intérêt; elle permet évidemment de mesurer la densité propre des rayons ligneux et d'estimer leur masse relative par rapport à celle du bois.

\section{3. - Mesure de la densité des gros rayons ligneux}

\section{3I. Matériel et méthode.}

Quinze échantillons pris parmi les soixante ont ainsi été sciés à $3 \mathrm{~mm}$ d'épaisseur et radiographiés (distance de la source au film : 2,50 $\mathrm{m}$; tension : $12 \mathrm{kV}$; intensité : $20 \mathrm{~mA}$; temps d'exposition : 25 minutes). Malheureusement, au moment où ces opérations ont été faites, nous ne disposions plus de l'analyseur de texture et

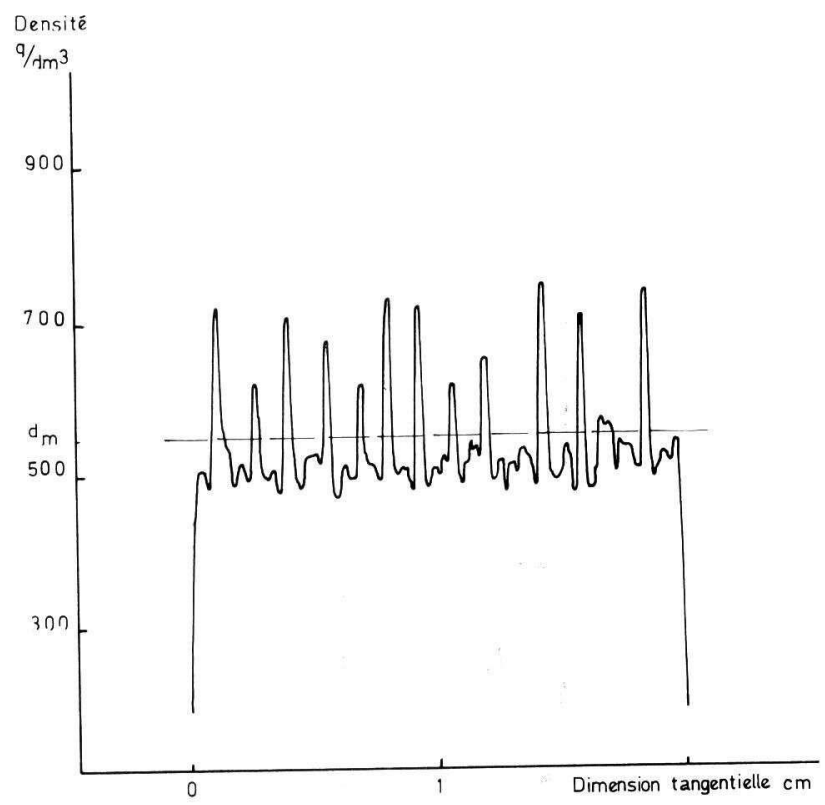

FIG. 6. - Profil densitométrique d'un échantillon analysé avec une fente large

On peut y dénombrer 22 pics correspondant aux gros rayons ligneux $d_{m}=$ densité moyenne $=546 \mathrm{~g} / \mathrm{dm}^{3}$

Densitometric profil of a sample which was analysed using a wide pencil of rays I 2 peaks, corresponding to the big wood rays, can be see $d_{m}=$ mean density $=546 \mathrm{~g} / \mathrm{dm}^{3}$ 
nous n'avons pu faire de nouvelles mesures de dénombrement et de proportions de rayons. La figure 5 met en évidence la différence de résolution de ces éléments entre la photographie et la radiographie du même échantillon, au profit incontestable de cette dernière dans l'éventualité d'études analogues ultérieures.

Les profils densitométriques ont été faits de deux façons : 1'une, en utilisant une fente exploratrice large de façon à intégrer le plus possible d'éléments dans le but de mesurer facilement une densité moyenne au planimètre; le faisceau analyseur avait dans ce cas tne largeur de $0,404 \mathrm{~mm}$ et une hauteur de I,34 $\mathrm{mm}$, et donc une section de $0,54 \mathrm{~mm}^{2}$. Dans cette modalité, seuls les très gros rayons ligneux sont bien individualisés sur les graphiques (fig. 6).

L'autre façon a consisté à utiliser au contraire une fente de faibles dimensions pour faire ressortir au maximum les rayons et obtenir même parfois des paliers sur les pies de densité correspondants. La présence d'un palier indique que la totalité du faisceau lumineux est à l'intérieur de l'image d'un rayon, et donc que l'ordonnée de ce palier est la densité propre des cellules le constituant. Pour obtenir ce résultat, on a choisi une fente de $0,026 \mathrm{~mm}$ de largeur sur $0,334 \mathrm{~mm}$ de hauteur, ce qui donne une section de $0,0087 \mathrm{~mm}^{2}$. Dans ces conditions, le profil de la figure 7 est constitué de la juxtaposition de très nombreux pics ; les plus bas sont dus partiellement aux

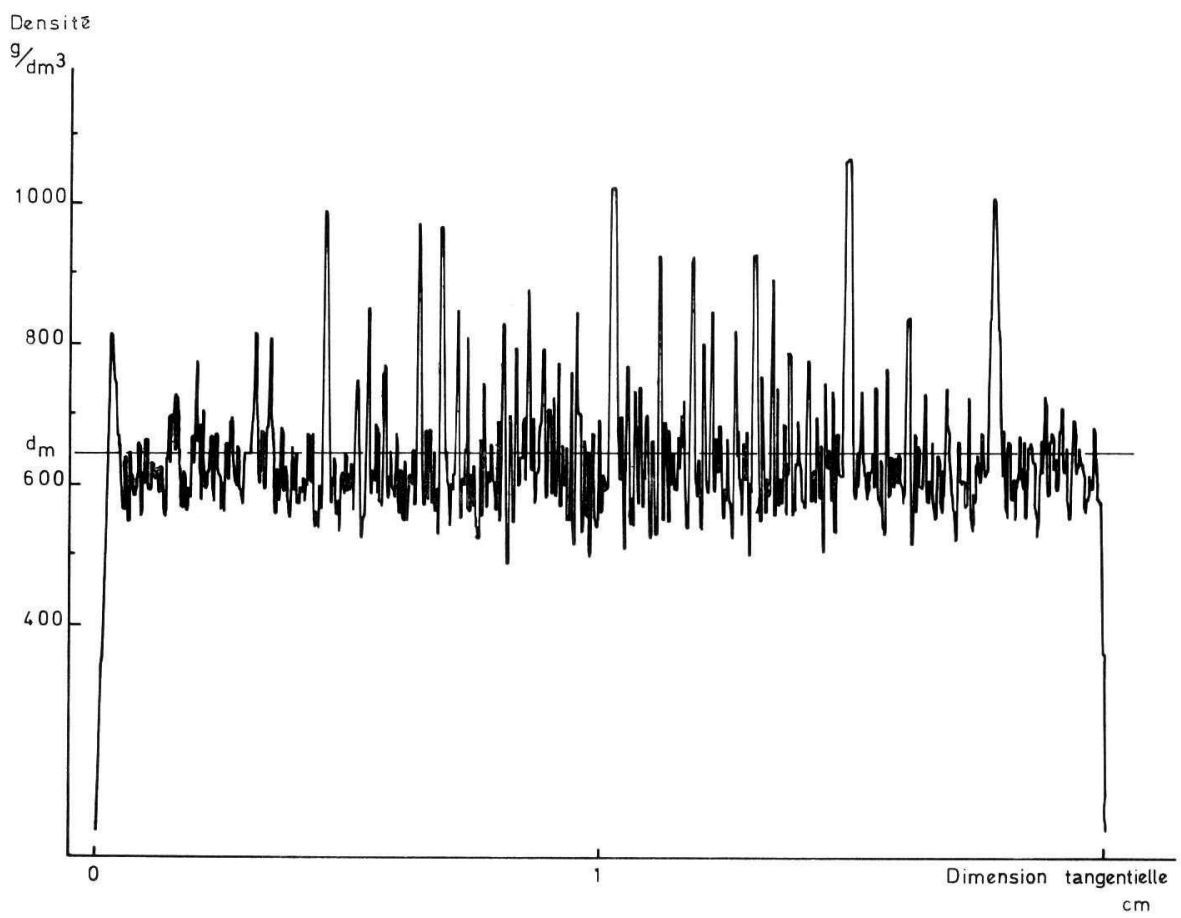

Fig. 7. - Profil densitométrique d'un échantillon analysé avec une fente étroite

La densité de certains rayons dépasse $\mathrm{I}$ ooo $\mathrm{g} / \mathrm{dm}^{3}$ $d_{m}=$ densité moyenne $=645 \mathrm{~g} / \mathrm{dm}^{3}$

Densitometric profil of a sample which was analysed using a narrow pencil of rays

The density of some wood rays is higher than $\mathrm{I}$ ooo $\mathrm{g} / \mathrm{dm}^{3}$ $d_{m}=$ mean density $=645 \mathrm{~g} / \mathrm{dm}^{3}$ 
très petits rayons, dont la largeur est du même ordre de grandeur que celle de la fente, et les plus hauts, aux gros rayons.

Pour estimer la valeur de la densité propre de ce tissu, on n'a retenu que les pics correspondant aux rayons dont la largeur réelle était supérieure ou égale à o, I mm à une ordonnée égale à celle de la densité moyenne du bois de l'échantillon considéré.

\section{32. - Résultats.}

Sur les quinze graphiques, on a trouvé I52 rayons satisfaisant à ces conditions. Le tableau 4 récapitule les résultats de leurs mesures :

\section{TABLEAU 4}

Densité des gros rayons ligneux et des échantillons les incluant

Density of the big reood rays and of the samples containing them

\begin{tabular}{|c|c|c|c|c|c|c|}
\hline Éléments mesurés & $\begin{array}{l}\text { Nombre } \\
\text { de mesures }\end{array}$ & $\begin{array}{l}\text { Minimum } \\
\left(\mathrm{g} / \mathrm{dm}^{3}\right)\end{array}$ & $\begin{array}{l}\text { Maximum } \\
\left(\mathrm{g} / \mathrm{dm}^{3}\right)\end{array}$ & $\begin{array}{l}\text { Moyenne } \\
\left(\mathrm{g} / \mathrm{dm}^{3}\right)\end{array}$ & $\begin{array}{l}\text { Ecart-type } \\
\quad\left(\mathrm{g} / \mathrm{dm}^{3}\right)\end{array}$ & $\begin{array}{c}\text { Coefficient } \\
\text { de variation } \\
(\%)\end{array}$ \\
\hline Gros rayons ligneux & 152 & 735 & 1080 & 898 & 64 & 7,1 \\
\hline $\begin{array}{l}\text { Profils densito- } \\
\text { métriques }\end{array}$ & 1.5 & $5 \times 7$ & $69 \%$ & $6 \cdot 29$ & 38 & 6,1 \\
\hline $\begin{array}{l}\text { éléments mesurés } \\
\text { nombre de mesur } \\
\text { gros rayons ligne } \\
\text { profils densitomé }\end{array}$ & $\begin{array}{l}\text { features } m \\
=\text { number } \\
=\text { big woo } \\
\text { pues }=\text { den }\end{array}$ & $\begin{array}{l}\text { sured } \\
\text { measureme } \\
\text { rays } \\
\text { tometric pro }\end{array}$ & & & & \\
\hline
\end{tabular}

La moyenne générale des observations est $8 \mathrm{~g} 8 \mathrm{~g} / \mathrm{dm}^{3}$ et, au seuil de confiance de 5 p. Ioo, elle peut varier entre $888 \mathrm{~g} / \mathrm{dm}^{3}$ et $909 \mathrm{~g} / \mathrm{dm}^{3}$.

La borne inférieure de la densité d'un pic est $735 \mathrm{~g} / \mathrm{dm}^{3}$ et la borne supérieure I $080 \mathrm{~g} / \mathrm{dm}^{3}$.

La densité moyenne du bois des quinze échantillons, déduite des mesures au planimètre, vaut $629 \mathrm{~g} / \mathrm{dm}^{3}$.

Par suite, le rapport $\mathrm{R}_{m}=\frac{\text { masse des rayons ligneux }}{\text { masse totale du bois }}$ est supérieur au rapport $\mathrm{R}_{v}=\frac{\text { volume des rayons ligneux }}{\text { volume total du bois }}$ puisque la densité propre des rayons, que l'on suppose en première approximation être la même pour les petits et les gros rayons, est très élevée. A partir de ces résultats, on peut calculer la densité des éléments du bois autres que les rayons, ainsi que le rapport $\mathbf{R}_{m}$.

Faisons ce calcul sur les chiffres moyens, d'une part en prenant $x$ égal à 0,04 , comme nous l'avons trouvé dans le cas des soixante échantillons, et d'autre part en admettant que les petits rayons qui n'ont pu être mis en évidence représentent un volume égal à celui des gros rayons, ce qui donne $x=0,08$. 
En effet, si $\rho \quad$ est la densité globale du bois

prl celle des rayons ligneux de toutes dimensions

parl celle des éléments autres que les rayons ligneux

$x=\mathrm{R}_{v}$, on peut écrire :

$$
\rho_{\text {arl }}=\frac{\rho-\rho_{r l} \cdot x}{I-x}
$$

où

$$
\rho=629 \mathrm{~g} / \mathrm{dm}^{3}, \rho_{r l}=898 \mathrm{~g} / \mathrm{dm}^{3} \text {. }
$$

Pour $x=0,04$, on trouve parl $=6 \mathrm{I} 7 \mathrm{~g} / \mathrm{dm}^{3}$ et $\mathrm{R}_{m}=0,06$

$$
x=0,08, \quad \text { parl }=606 \mathrm{~g} / \mathrm{dm}^{3} \text { et } \mathrm{R}_{m}=0, \mathrm{II} .
$$

\section{5. - CONCLUSION}

L'utilisation conjuguée de la radiographie et d'appareils capables d'analyser la texture d'échantillons serait, semble-t-il, bien adaptée à l'étude du rôle des rayons ligneux dans la qualité du bois du hêtre.

On a pu mettre en évidence par cette méthode que ces éléments anatomiques denses ont leur importance dans les propriétés physiques et mécaniques. Ils ont une action favorable si l'on se place du point de vue d'une utilisation réclamant de fortes résistances aux contraintes, mais défavorable vis-à-vis des propriétés de rétractibilité radiale et tangentielle.

Cette étude confirme aussi l'intérêt des résultats obtenus sur les jeunes plants de hêtre (H. POLGE et al., I972 op. cit.) chez qui ont pu être mis en évidence les effets du sol et de l'hérédité sur diverses caractéristiques, dont le nombre de rayons ligneux.

Si ce dernier, qui est en corrélation positive avec l'infradensité totale, est trop élevé, le bois risque d'être trop dense, donc trop dur, même si par ailleurs il a une bonne porosité, et sa qualité en est affectée, surtout dans 1'hypothèse de certains emplois, tels que le déroulage ou la menuiserie fine.

Ces indications suggèrent d'entreprendre une étude dans laquelle les propriétés du bois de hêtre seraient appréciées à partir de la densité globale d'une part, et de la densité calculée sur les éléments autres que les rayons ligneux d'autre part.

Il est certain qu'un procédé de mesure automatique globale du type de celui qu'on a utilisé, en permettant de faire des essais sur de nombreux échantillons, pourrait aider à mieux connaître ces tissus, en particulier leur variabilité et leur abondance en fonction des individus et du milieu, et à estimer des paramètres génétiques. Cela compléterait les études sur la qualité du bois qui, jusqu’ici, les ignoraient presque toujours.

Rę̧u pour publication en février 1975.

\section{SUMMARY}

THE EFFECT OF THE BIG WOOD RAYS UPON SOME PROPERTIES OF BEECH WOOD

The big wood rays of standardized samples of beech (fagus silvatica) have been automatically numbered and measured by a texture analyser working on photographs of their tangential plane. 
The comparison of these results and the results of mechanical and physical tests carried out on the samples, shows that the wood rays are factors of high mechanical strength and high tangential shrinkage.

It would appear that the use of radiography instead of photography leads to better resolution by the texture analyser; it also gives a means of estimating, as we did, the value of wood ray density (in $\mathrm{g} / \mathrm{dm}^{3}$ ) and afterwards, of differentiating between the properties associated with the rays and those associated with other wood elements.

\title{
ZUSAMMENFASSUNG
}

\author{
DER EINFLUSS DER BREITEN MARKSTRAHLEN \\ AUF EINIGE EIGENSCHAFTEN VON BUCHENHOIZ
}

Die breiten Markstrahlen an normalisierten Probestäben von Buchenholz (Fagus silvatica) wurden mittels eines Bildanalysators automatisch gezählt und gemessen und zwar auf Grund der Photographischen Aufnahme ihres Tangentialschnitt.

Vergleicht man die Daten des Analysators mit den Ergebnissen der mechanischen und physischen Messungen dieser Probestäbe so erweist es sich, dass die Markstrahlen Faktoren einer hohen mechanischen Festigkeit sind, sowie einer beträchtlichen Tangentialschwindung.

Die Anwendung der Röntgenaufnahme an Stelle der Photographie würde es dem Analysator eine noch grössere Präzision zu erreichen; die Röntgenaufnahme wurde übrigens angewendet, um den eigentlichen Wert der Dichte $\left(\mathrm{g} / \mathrm{dm}^{3}\right)$ dieser anatomischen Elemente $\mathrm{zu}$ messen, und dann die Eigenschaften die den Markstrahlen zuzuschreiben sind, von denjenigen zu unterscheiden, die mit anderen Elementen in Verbindung stehen.

\section{RÉFÉREINCES BIBLIOGRAPHIQUES}

Boutelje J. B., I962. The relationship of structure to transverse anisotropy in wood with reference to shrinkage and elasticity. Holzforschung, 16 (2), 33-46.

JAYMe G., Azzola F. K., 1965. Licht - und elektronenmikroskopische Beobachtungen an Rotbuchen - Längsparenchym (Fagus silvatica L.). Holzforschung, 19 (I), I-4.

KEnNedy R. W., I968. Wood in transverse compression. Influence of some anatomical variables and density on behavior. Forest Products Journal, 18 (3), 36-40.

Koltzenburg C., I966. Die Abhängigkeit der Holzeigenschaften der Rotbuche (Fagus silvatica L.) von Lichtgenuss, soziologischer Stellung und anderen Wuchsbedingungen. Dissertation zur Erlangung des Doktorgrades der Forstlichen Fakultät der Georg-August-Universität zu Göttingen in HannMünden.

Myer J. E., I930. The structure and strength of four North American woods as influenced by range habitat, and position in the tree. Bulletin of the New York State College of Forestry at Syracuse Univer sity. Technical Publication $\mathrm{n}^{\circ} 3 \mathrm{I}$.

Panshin A. J. C., de Zeeuw C., Brown H. P., I964. Textbook of teood technology. Second edition, McGraw-Hill Book Company, New York.

Polge H., Keller R., Thiercelin F., Garros S., I972. Effets du sol et de l'hérédité sur la croissance et les caractéristiques anatomiques de jeunes plants de hêtre. Document à distribution limitée Station de Recherches sur la Qualité des Bois, C. N. R. F., no 72-I.

SAchsse H., I974. Eine Anomalie des Strahlgewebes von Fagus sylvatica L. Holz als Roh- und Werkstoff, $32^{\mathrm{e}}$ année, cahier 3, 95-98.

Schniewind A. P., I959. Transverse anisotropy of wood a function of gross anatomic structure. Forest Products Journal, 9 (I0), 350-399.

Schniewind A. P., I966. Irregularities of finished surfaces caused by unequal ray shrinkage. Forest Products Journal, 16 (8), 66-67.

Thiercelin F., Perrin J. R., ig7z. Débit d'échantillons d'épaisseur rigoureusement uniforme à l'aide de scies-fraises jumelées. Annales des Sciences forestiores, 30 (2), 295-299. 
TAYLOR F. W., I969. The effect of ray tissue on the specific gravity of wood. Wood and Fiber, 1 , été $1969,(2), 142-145$.

TAYLOR F. W., Wooten T. E., 1973. Wood property variation of Mississipi delta hardwoods. Wood and Fiber, 5 , printemps I973, (I), I-I3.

Tellerup E., de Muckadell M. S., I953. Individual differences in the shape of wood rays in Fagus sylvatica L. A wood anatomical investigation. Royal Veterinary and A gricultural College, Copenhague, Yearbook I953, 147-157.

WAGENFÜHR R., I966. Anatomie des Holzes. VEB Fachbuchverlag, Leipzig. 